\title{
The Impacts of Tumorigenic and Nontumorigenic Agrobacterium vitis Strains on Graft Strength and Growth of Grapevines
}

Lingyun Hao, College of Life Sciences and Oceanography, Shenzhen University, Guang Dong 518060, P.R. China, and Section of Plant Pathology and Plant-Microbe Biology, SIPS, Cornell University-New York State Agricultural Experiment Station, Geneva, NY, 14456; David J. Kemmenoe, Sibley School of Mechanical and Aerospace Engineering, Cornell University, Ithaca, NY 14850; Didem Canik Orel, Department of Plant Protection, Ankara University, Ankara, Turkey; and Thomas Burr, ${ }^{\dagger}$ Section of Plant Pathology and Plant-Microbe Biology, SIPS, Cornell University-New York State Agricultural Experiment Station

\begin{abstract}
The effects of tumorigenic and nontumorigenic strains of Agrobacterium vitis on graft strength and growth of grapevines was studied. A procedure was developed for inoculating graft interface surfaces with A. vitis and for measuring the force required to break grafts at different time points. Cuttings were soaked in an aqueous suspension of bacteria, about $10^{6} \mathrm{CFU} / \mathrm{ml}$, and bacteria were spread onto the graft interface during the grafting procedure. Tumorigenic strain CG49 caused reduced bud germination and increased callus (crown gall) at the graft union and at the base of cuttings

at 30 days postinoculation (dpi) and significantly reduced shoot growth by 60 dpi whereas, at the same time points, nontumorigenic strain F2/5 inhibited callus formation but did not affect bud germination or shoot growth. Graft strength was enhanced at $30 \mathrm{dpi}$ with CG49, presumably because the crown gall callus served to secure the union; graft strength was weakened by F2/5 over the same period. Between 30 and $60 \mathrm{dpi}$, the greatest increase in graft strength was observed in the water control. Following graft union inoculations, the A. vitis population increased more than 1,000-fold within 5 days.
\end{abstract}

Grafting is a widely used vegetative propagation technique employed in grape nursery production worldwide. It involves connecting a scion variety to a rootstock to generate a unified plant, and depending on rootstock provides desirable traits to the vine such as Phylloxera and nematode resistance, salt tolerance, and vine vigor control (Alleweldt and Possingham 1988). Successful graft compatibility requires the generation of a functional vascular system between the scion and the rootstock resulting in a viable plant that is able to sustain optimum growth and productivity. Incompatibility, or inferior graft take, due to poor vascular connection and phloem regeneration at the graft union can result in reduced water and nutrient flow, leading to poor plant development and even death (Milien et al. 2012). Genetic, biotic, physiological, and environmental factors all play roles in graft success; these may include genetic compatibility between the scion and rootstock variety, maturity of the grafting material, and environmental conditions during growth of the propagation material and during the grafting process (Cookson et al. 2013; Tandonnet et al. 2010). Plant pathogens can have significant impacts on graft take and subsequent plant development (Fourie and Halleen 2006).

Agrobacterium vitis is a bacterial pathogen that is host specific to grapevines (Vitis spp.). It causes crown gall (tumor formation) disease which can result in reduced plant growth and productivity that may end in vine decline and death (Burr et al. 1998). The infection process of $A$. vitis is similar to that of $A$. tumefaciens, which is initiated at plant wound sites and involves transfer, insertion, and expression of a section of the tumor-inducing plasmid, the transfer DNA (T-DNA), into the plant chromosome (Schell and Van Montagu 1977). Grafting of grapevines provides a significant wound site at which A. vitis can initiate infection. We previously developed a highly sensitive method for detecting tumorigenic A. vitis in grapevines (Johnson et al. 2013). The pathogen was detected in all parts of the vines, including nodes, internodes, shoot tips, and leaves (Johnson et al. 2016; Orel et al. 2017). Because the bacterium persists systemically and epiphytically in vines, it may become established on graft surfaces during the grafting process.

${ }^{\dagger}$ Corresponding author: T. Burr; E-mail: tjb1@ cornell.edu

Accepted for publication 30 September 2017.

(c) 2018 The American Phytopathological Society
Currently, there is no effective chemical control for grape crown gall, and other management strategies such as varietal selection and cultural practices are only partially effective. In recent years, research has focused on the development of biological control agents to suppress grape crown gall, and several candidate strains have been reported, including nontumorigenic A. vitis strains E26, VAR03-1, and ARK-1 (Kawaguchi 2013; Kawaguchi and Inoue 2012; Kawaguchi et al. 2008), and strains of other bacterial genera, including Rahnella aquatilis strain HX2 (Chen et al. 2007), Serratia plymuthica strain IC1270, Pseudomonas fluorescens strain Q8r1-96, P. fluorescens strain B-4117 (Dandurishvili et al. 2011), and Bacillus subtilis strain 14B (Hammami et al. 2009). These strains have shown various levels of disease suppression in experiments done under greenhouse conditions. Our lab has studied a nontumorigenic strain, F2/5 that suppresses crown grape crown gall (Staphorst et al. 1985) and we demonstrated inhibition of tumorigenicity by diverse $A$. vitis strains on different grape varieties (Burr and Reid 1994). However, like other tumorigenic and nontumorigenic $A$. vitis strains, F2/5 induces localized necrosis on grape tissues that would hinder its development for commercial application (Burr et al. 1987). The molecular mechanism involved in necrosis is not fully understood but includes quorum-sensing regulation and the involvement of different polyketide and nonribosomal peptide synthases (Hao et al. 2005; Zheng and Burr 2013; Zheng et al. 2003, 2012). Our current understanding of the biochemical pathways associated with tumor inhibition by F2/5 and necrosis show overlap but also distinct processes (Zheng and Burr 2016) and are still under investigation.

For the work reported in this article, we sought to determine how the presence of $A$. vitis at grape graft unions affects graft strength and subsequent plant development. Because crown gall in nurseries and in young vineyards is frequently first observed at graft unions, we hypothesize that $A$. vitis, which is distributed randomly in and on grapevines, affects graft strength, resulting in reduced graft take. Several methods have been used to measure the physical properties of graft unions such as hydraulic conductance measurement, microscopy, and tensile strength testing (Atkinson et al. 2003; Moore 1983; Zarrouk et al. 2010). Tensile strength in tomato plants has been reported primarily as force divided by grafted area, with force being applied by hand and measured with a pull-force gauge (Bausher 2013; Lindsay et al. 1974; Moore 1983; Parkinson and Yeoman 1982). This is a good first estimate of strength but may not work well for stronger grafts such as grafted grapevines or other woody plants. Pedersen 
measured the tensile strength of cherry tree grafts with a traditional tensile test machine (Pedersen 2005), although they were limited by issues with gripping graft samples and load cell maximum load. For this work, we develop a method to quantitatively measure graft strength which is appropriate for both low and high force measurements while being rapid enough to test the large number of samples necessary for statistical interpretation.

In this study, we investigated the effects of a tumorigenic (CG49) and a nontumorigenic (F2/5) A. vitis strain on grape graft strength and subsequent graft take and plant development by comparing callus and root formation, bud germination, and shoot growth in addition to graft strength. These data advance our understanding of the epidemiology of A. vitis and will be particularly relevant to the grape nursery industry and for the future commercial development of effective controls for grape crown gall.

\section{Materials and Methods}

Strains, culture conditions, and plant materials. Tumorigenic strain CG49 and nontumorigenic strain F2/5 were maintained at $28^{\circ} \mathrm{C}$ on potato dextrose agar (PDA) plates (Difco Laboratories, Detroit). F2/5 has been studied previously for its ability to inhibit the development of crown gall on grapevines (Burr and Reid 1994). The F2/5 Davi4329 strain (Zheng and Burr 2016) was cultured sim-

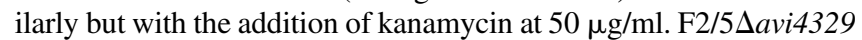
was made by disrupting gene $F$-avi4329, which encodes an aminotransferase. The mutant behaves identically to wild-type F2/5 in its growth and ability to inhibit crown gall formation on grape Dormant canes of Vitis vinifera 'Cabernet Franc', a crown gall-susceptible variety, were collected in early spring 2016 from a research vineyard at Cornell's New York State Agricultural Experiment Station in Geneva, NY and stored at $4{ }^{\circ} \mathrm{C}$.

Indexing dormant cuttings for presence of tumorigenic $A$. vitis. Forty dormant Cabernet Franc cuttings made from the canes mentioned above were randomly chosen and assayed for the presence of tumorigenic $A$. vitis strains. In total, three nodes were sampled for each cutting and extraction of potential A. vitis cells from cuttings and subsequent enrichment was done as previously described (Johnson et al. 2013). A magnetic capture hybridization (MCH) method was used to extract the target DNA followed by real-time polymerase chain reaction (PCR) to detect the presence of tumorigenic A. vitis (Johnson et al. 2013).

Inoculation of dormant cuttings. Stored dormant Cabernet Franc cane sections were cut into single-node cuttings (with bud near the apical end) of about 70 to $80 \mathrm{~mm}$ in length and soaked in water at room temperature overnight to hydrate buds. The cuttings were then soaked in different concentrations of aqueous bacterial cell suspensions (CG49 or F2/5 for $2 \mathrm{~h}$ at $1 \times 10^{5}, 1 \times 10^{6}$, or $1 \times 10^{7} \mathrm{CFU} / \mathrm{ml}$ ). Bacterial cell suspensions were made by initially preparing a suspension at an optical density at $600 \mathrm{~nm}\left(\mathrm{OD}_{600}\right)=0.1$ (about $1 \times$ $10^{8} \mathrm{CFU} / \mathrm{ml}$ ) and making appropriate dilutions. Populations were confirmed by dilution plating the initial $\mathrm{OD}_{600}=0.1$ suspensions on PDA plates. Plants soaked in water alone were included as the control group. A hand-held grafting tool (A. M. Leonard, Piqua, $\mathrm{OH}$ ) was used to make a $\mathrm{V}$-shaped cut through the midpoint of each cutting, during which the bacteria on the outer cutting surface were spread onto the graft wound surfaces. The upper and lower sections were then sealed together as an autograft with melted grafting wax. By initially soaking cuttings in the bacterial suspensions, wounds at the basal and apical ends of the cuttings were also inoculated. Tools and bench surfaces were surface disinfected with $70 \%$ ethanol between treatments to avoid cross-contamination; 30 to 50 grafts were included for each treatment in each round of experiment, depending on the assay. Grafted cuttings were planted in moist perlite and pots were covered with plastic wrap to maintain high humidity until buds germinated (about 10 days) at room temperature; then, the plastic wrap was removed and grafted cuttings were transferred into the greenhouse and maintained at $28^{\circ} \mathrm{C}$. The surface of the perlite was watered lightly every few days to keep it moist. At 30 days postinoculation (dpi), specific phenotypes were recorded for each treatment, as described below.
Population dynamics of $\boldsymbol{A}$. vitis in graft wounds. The population dynamics of $A$. vitis in graft wounds was assessed using strain F2/ $5 \Delta$ avi4329 (Zheng and Burr 2016) because it allowed plating on media amended with kanamycin. Cuttings were soaked in a bacterial suspension of about $1 \times 10^{6} \mathrm{CFU} / \mathrm{ml}\left(1: 100\right.$ dilution of an $\mathrm{OD}_{600}=$ 0.1 ) and grafting (inoculation of graft surfaces) was done with the V-graft tool, as described above. Grafts were planted in perlite and populations on the $\mathrm{V}$-cut surface were determined at $0,5,18,30$, and $60 \mathrm{dpi}$ The grafted cuttings were first surface swabbed with $70 \%$ ethanol, followed by rinsing with sterile distilled water (SDW), and then separated at the graft union by pulling them apart. One-half of the upper graft section (one-quarter of the total graft union area) was then debarked and separated from the rest of the graft area with a sterile razor blade and placed in $1 \mathrm{ml}$ of SDW in an Eppendorf tube. Tubes were vigorously vortexed for $15 \mathrm{~s}$ to release bacterial cells on the graft union surface; serial dilutions were plated onto PDA plates supplemented with kanamycin and incubated at $28^{\circ} \mathrm{C}$ for about $40 \mathrm{~h}$, at which time colonies were counted. Razor blades were disinfected with $70 \%$ ethanol between each sample to avoid cross contamination. Eight replicates were included for each treatment at each time point, and the experiment was repeated twice.

Bud germination. At $30 \mathrm{dpi}$, cuttings that were inoculated with CG49, F2/5, or water were harvested, and the percentage of buds that germinated for each treatment was determined. For a subset of plants that developed a growing bud, data were recorded on callus formation, plant biomass, and graft strength. The remaining plants with developing shoots and roots were transplanted in pots containing a greenhouse mix and kept for another 30 days, at which time the same set of traits was measured. Plants were watered periodically to keep the soil moist.

Callus formation and root and shoot biomass development. At 30 and 60 dpi, plants inoculated with CG49, F2/5, or water were harvested to observe external callus formation at the graft union and on the basal and apical ends. No callus was ever apparent at apical ends and, therefore, callus formation at the graft union and the basal end of cutting was rated based on a 1-to-4 or 1-to-6 scaling system, respectively, with a rating of 1 for grafts with minimum callus formation only found on one side of the V-graft area to 4 for those with strongest callus formation on both sides of the cut. Similarly, callus formation on the basal surface of the cuttings was rated following a 1-to-6 scaling system based on size of callus in relation to the total basal surface area, where $1=$ less than half of the surface area, $2=$ half of the surface area, $3=$ more than half but less than the whole surface area, $4=$ more than the whole but less than twice of the surface area, $5=$ twice of the surface area, and $6=$ three-dimensional callus formation. If no obvious callus formation was observed, samples were given a rating of 0 . Total root biomass and shoot biomass were also measured. Root and shoot development was recorded by individually weighing the total biomass. At least 18 plants were included for each treatment at each time point. The root biomass evaluation was repeated twice and all other experiments were repeated three times.

Graft strength measurement. An Instron 5569 load frame with a $5 \mathrm{KN}$ load cell (Instron, Norwood, MA) was used to apply 3-pt bending loading to grafted specimens (Fig. 1). The span was set to $40 \mathrm{~mm}$ with the graft in the center and force applied through a $3 \mathrm{~mm} / \mathrm{min}$ downward displacement rate. The displacement was continued until there was a clear decrease in load (indicating graft breakage) and maximum load was recorded. Graft strength was determined as the maximum force that was needed to break each grafted cutting. Prior to doing the graft strength test, the diameter of each graft was measured using digital metric calipers; two diameter readings rotated $90^{\circ}$ were averaged and the average was recorded. This was repeated on either side of the graft and the reported diameter measurements were calculated by averaging the average value of either side of the graft. This measurement was taken to help account for thickness differences between cuttings. Where necessary, graft nodes were cut off with a pruning knife to produce flat segments to maintain the graft positioning on the fixture

Statistical analysis. All statistical analyses were performed using the $\mathrm{R}$ program. To compare the mean failure rate of early bud germination between treatments, a one-tailed Student's $t$ test was performed. 
To compare mean callus formation and fresh root or shoot biomass among treatments, a one-way analysis of variance and post hoc comparisons between treatments were performed using Tukey's honestly significant difference test to correct for multiple comparisons. To compare the mean graft strength between treatments, a linear mixed-effects model was used to include fixed effects of graft diameter and treatment and random effects to capture the variability between the experiments. A residual analysis was performed to assess the validity of the assumptions of normality and homogeneous variances. A $P$ value $<0.05$ was considered to be significant.

\section{Results}

Indexing of dormant canes for presence of tumorigenic $A$. vitis. As indicated above, an MCH real-time PCR method was used to assay the Cabernet Franc cuttings used in this research to determine the incidence of $A$. vitis in them (Johnson et al. 2013). In all, 14 of 40 cuttings tested positive for $A$. vitis (data not shown). Although specific population levels of $A$. vitis in the samples were not determined, it was previously shown that $A$. vitis is randomly distributed in cuttings and that the MCH method is able to detect as few as 10 cells per sample (Johnson et al. 2013, 2016).

Dose effect of bacteria on necrosis and callus formation. We compared the effect of three levels of bacterial cells of F2/5 and CG49 on necrosis and callus development at basal ends of the grafted

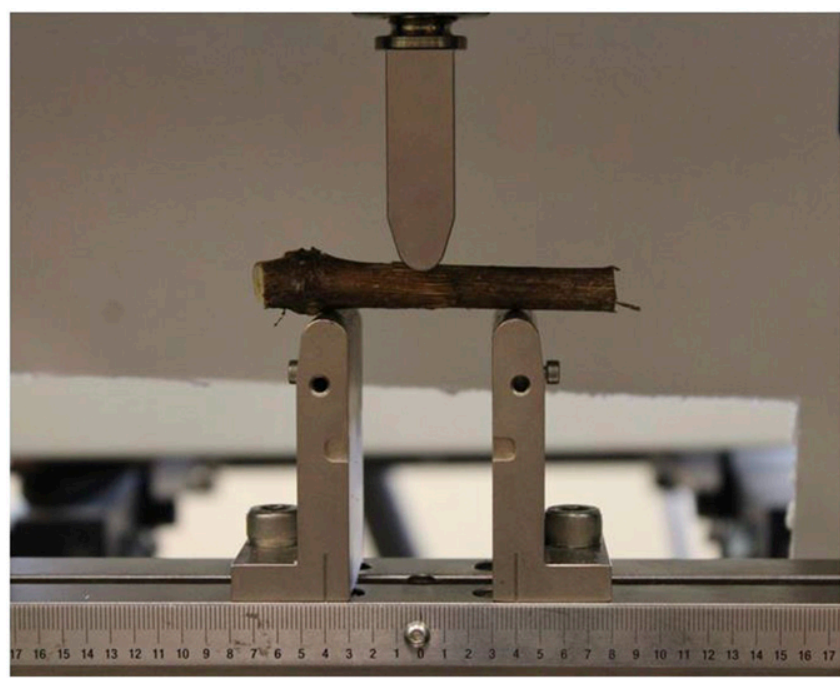

Fig. 1. Grafted cutting positioned on the testing platform of the Instron 5569 machine. cutting. At 30 dpi, 9.4, 26.1, and $60 \%$ of plants inoculated with $1 \times 10^{5}, 1 \times 10^{6}$, or $1 \times 10^{7} \mathrm{CFU} / \mathrm{ml}$ of CG49 cells, respectively, developed necrosis, whereas none or minimal necrosis was evident on the water-treated plants (Fig. 2B; Table 1). Thus, the necrosis level that developed at the basal ends correlated with the level of bacterial inoculum. Similar results were observed with F2/5, for which reduced callus formation and increased necrosis occurred at basal ends with the higher concentrations of inoculum. No necrosis or callus was ever observed at the apical cut ends of the cuttings treated with either bacteria. CG49 induced the strongest callus formation (expressed as crown gall) at graft unions when inoculated with $1 \times 10^{6} \mathrm{CFU} / \mathrm{ml}$ of bacteria (Fig. 2B). It may be that, at the $1 \times 10^{7} \mathrm{CFU} / \mathrm{ml}$ level of CG49, increased necrosis formed restricting callus (crown gall) formation. Similarly, strain F2/5 may reduce callus formation by necrosisinduced death of cambial cells and by its inherent ability to inhibit crown gall on grapes (Creasap et al. 2005). Although natural populations of $A$. vitis at individual graft and other wound sites are likely to vary, we selected an initial cutting soaking inoculum concentration of $1 \times 10^{6} \mathrm{CFU} / \mathrm{ml}$, which we calculated would deliver about 500 bacterial cells to the graft union surface (as shown below) for subsequent experiments. Our reasoning was to use an inoculum level that is relatively low, likely to be found naturally in plants, and would allow plants to be maintained for an extended term for assessments of growth and phenotypes such as callus formation at graft unions.

Population dynamics of $\boldsymbol{A}$. vitis at the grape graft union. Initially, it was shown that in vitro growth of F2/5 $\Delta$ avi4329 does not differ from CG49 or F2/5 (data not shown) and, thus, it could be used to estimate the population dynamics of A. vitis on graft surfaces. Subsequently, experiments were done to determine how the inoculum concentration used for soaking cuttings translated to bacterial numbers deposited on the graft surface and how populations changed over time. Approximately 1.6, 2.7, and $3.7 \log$ units of F2/5 $\Delta$ avi4329 were introduced to graft surfaces when inoculum at $1 \times 10^{5}, 1 \times 10^{6}$, and $1 \times 10^{7} \mathrm{CFU} / \mathrm{ml}$, respectively, was used (Fig. 2A). Therefore our results showed a strong correlation between inoculum concentration and the initial populations deposited on the graft surface, as would be predicted if the bacterium was spread to the graft surface during the grafting process. Growth of $A$. vitis on the surface of graft unions was determined up to $60 \mathrm{dpi}$. About 500 bacterial cells were detected on the graft surface immediately following inoculation (Fig. 3). Thus, it was possible to introduce a repeatable level of inoculum, similar to that in Figure 2A, through the graft inoculation method. F2/5 4329 grew rapidly at the graft surface, with a population increase of more than a 1,000-fold (from 2.7 to $6.0 \log$ units), within 5 dpi. Bacterial growth slowed from day 5 to 18 , with a population increase

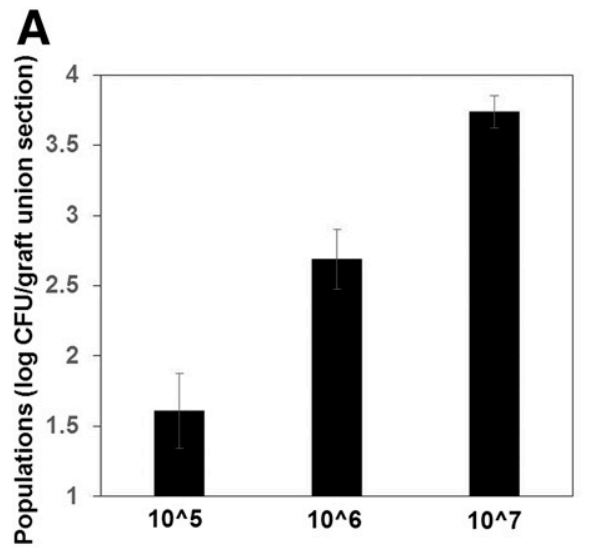

B

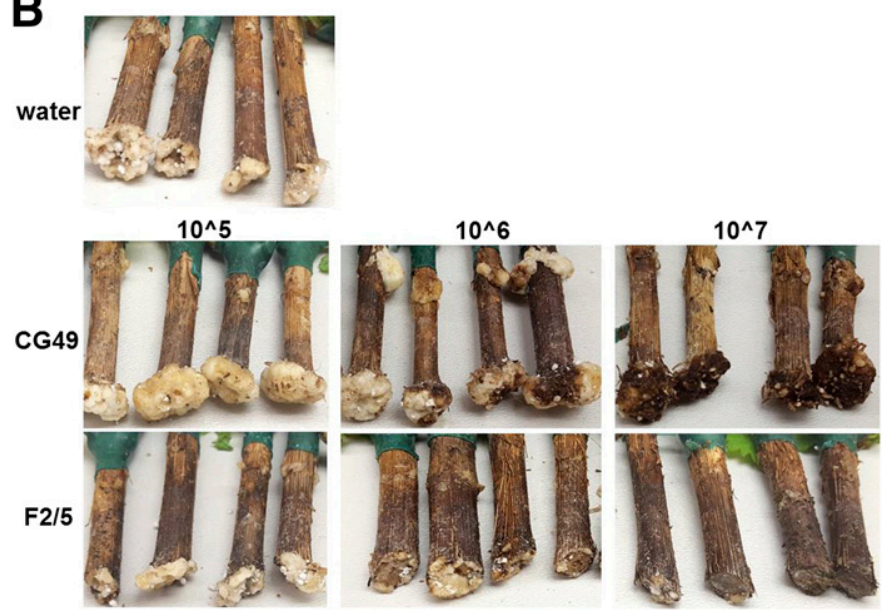

Fig. 2. Effect of inoculum dose on cell numbers of Agrobacterium vitis inoculated to graft union surfaces and the effect of the bacterium on necrosis and callus formation. A, Mean $\log$ units of $\mathrm{F} 2 / 5 \Delta$ avi4329 cells at 0 -time that were deposited by the grafting blade on the graft union surface when $10^{5}$, $10^{6}$, and $10^{7} \mathrm{CFU} / \mathrm{ml}$ were used as primary inoculum. Eight replicates were included for each treatment at each time point and the experiment was repeated twice. Error bars are standard deviations. B, Necrosis and callus formation on cutting base and graft union inoculated with water, CG49, or F2/5. 
of approximately $0.5 \mathrm{log}$ units, and was maintained at the level of $6.6 \log$ units through day 60 , at which time the experiment was concluded. These results indicated that, once introduced into a graft wound site, A. vitis grows rapidly and maintains a high population level for an extended period of time. Thus, further wounding at a graft union from partial breakage may initiate crown gall infections.

Impact of CG49 and F2/5 on bud germination and survival. After 30 days, $7 \%$ of the buds on plants in the control group (treated with water) did not germinate. In contrast, plants treated with CG49 had the lowest bud germination rate, with a $25.6 \pm 6.3 \%$ failure rate ( $P<0.01$, compared with control group) (Fig. 4). For F2/5-treated plants, $12.9 \pm 5.2 \%$ of the buds did not germinate, which represents a slight difference from that of the control $(0.05<P<0.1)$. Therefore, treating plants with tumorigenic strain CG49 at $10^{6} \mathrm{CFU} / \mathrm{ml}$ had a clear negative impact on bud germination at 30 days, whereas F2/5 showed a greater number of ungerminated buds than the control but the difference was not statistically significant. The initial inoculum concentration also effected the level of germinated buds that subsequently collapsed following transplanting (at $60 \mathrm{dpi}$ ). For plants treated with $10^{6} \mathrm{CFU} / \mathrm{ml}, 16 \%$ percent of the germinated buds collapsed and, for $10^{7} \mathrm{CFU} / \mathrm{ml}, 30 \%$ collapsed. For the water treatment, there was no germinated bud collapse. Each treatment consisted of 30 to 38 plants. Therefore, concentration of inoculum used to inoculate plants had a significant effect on bud germination and survival. From these experiments, it is not possible to determine whether graft inoculation or inoculation at basal or apical ends or the external surface of the buds affected their germination.

Effects of CG49 and F2/5 on callus formation at cutting base and graft unions. External callus formation was visually assessed

Table 1. Dose effect of Agrobacterium vitis strains CG49 and F2/5 on the percentage of grafted grape plants with basal necrosis 30 days postinoculation

\begin{tabular}{lcc}
\hline Treatment & $\begin{array}{c}\text { Inoculum } \\
\text { (CFU/ml) }^{\mathbf{a}}\end{array}$ & $\begin{array}{c}\text { Plants with obvious necrosis } \\
\text { at cutting base } \mathbf{( \% )}\end{array}$ \\
\hline CG49 & $10^{5}$ & 9.4 \\
& $10^{6}$ & 26.1 \\
F2/5 & $10^{7}$ & 60.0 \\
& $10^{5}$ & 23.7 \\
& $10^{6}$ & 31.4 \\
Water & $10^{7}$ & 59.1 \\
\hline
\end{tabular}

${ }^{\mathrm{a}}$ Inoculum level in which dormant cuttings were soaked prior to grafting. NA = not applicable.

${ }^{b}$ Based on a total of 52 to 56 grafts for each treatment.

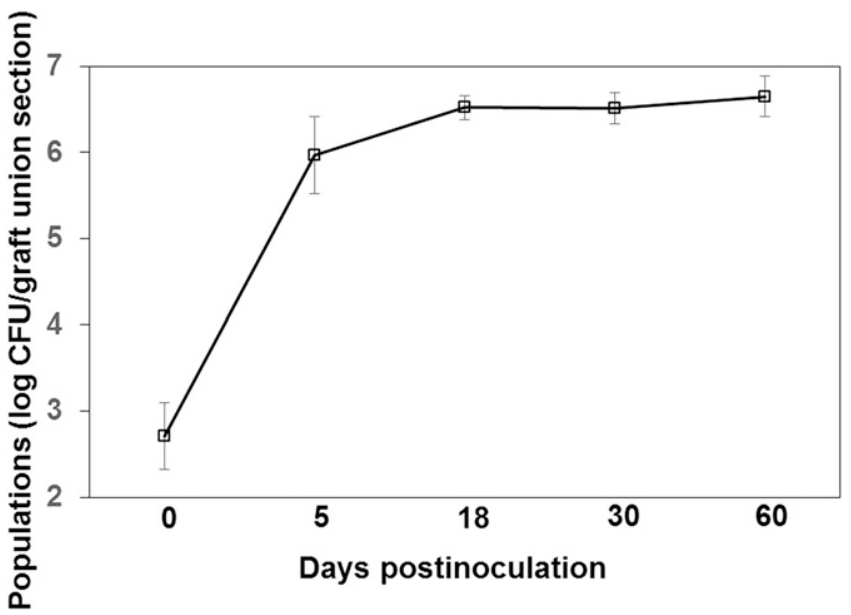

Fig. 3. Population dynamics of F2/5 $\Delta a v i 4329$ at inoculated graft unions. Shown are mean log units of populations on graft union surface at $0,5,18,30$, and 60 days postinoculation, respectively. Eight replicates were included for each treatment at each time point and the experiment was repeated twice. Error bars are standard deviations. immediately after harvest at 30 dpi using scaling systems (Fig. 5A). CG49-inoculated plants had significantly more callus (crown gall) at both the basal ends and graft unions, with an average rating of 4.0 and 1.4 respectively, compared with an average rating of 3.4 $(P<0.01)$ and $0.4(P<0.01)$ in the water treatment (Fig. 5B). In contrast, strain F2/5 greatly inhibited callus formation at the cutting base, resulting in a lower average rating of 2.1 compared with the water treatment $(P<0.01)$. Both $\mathrm{F} 2 / 5$ and water treatments showed a low level of external callus formation at the graft unions at the time intervals used in these experiments, with no difference observed between them $(P>0.1)$.

Similar results were observed in plants harvested at $60 \mathrm{dpi}$; CG49 induced the greatest level of callus (crown gall) on both base of cuttings $(P<0.01$ compared with both water and $\mathrm{F} 2 / 5$ treatments) and at graft unions $(P<0.01$ compared with both water and $\mathrm{F} 2 / 5$ treatments), and F2/5 induced the least callus formation on cutting base but no difference from the water treatment at graft union (Fig. 5C).

Impact of CG49 and F2/5 on root and shoot development of grafted cuttings. Root development at 30 dpi was quite variable between samples, yielding no significant difference in fresh root biomass among the three treatments (data not shown). Similar observations were seen again at $60 \mathrm{dpi}$ and, thus, no further quantification of fresh root biomass was taken for additional experiments. However, shoot growth on CG49-treated plants was significantly less at 60 dpi (Fig. 6A), with an average (Avg) fresh shoot biomass of approximately $6.3 \mathrm{~g}$, which was significantly less than that of the F2/5 (Avg $=9.2 \mathrm{~g}$, $P<0.01)$ or water treatments (Avg $=10.5 \mathrm{~g}, P<0.01)$ (Fig. 6B). Therefore, CG49 caused a reduction in plant growth at 60 dpi whereas F2/5 had a reduced but not statistically significant amount of shoot growth. As for the bud germination experiments, it is not possible to discern whether the reduction of shoot growth is a result of $A$. vitis at the graft union (i.e., inferior union formation) or from the bacterium causing extensive callus or necrosis or other effects at the base or apical ends of the cutting.

Impact of CG49 and F2/5 on graft strength. During preliminary investigations on the effect of $A$. vitis on graft strength, we observed that inoculation with higher cell numbers resulted in grafts that were easily broken by hand at 30 dpi. To quantify the effect of $A$. vitis, we used an Instron 5569 machine to measure the graft strength, as described in the methods above. At 30 dpi, CG49-treated plants had higher average graft strength $(\mathrm{Avg}=34.6$ Newtons $[\mathrm{N}])$ than that

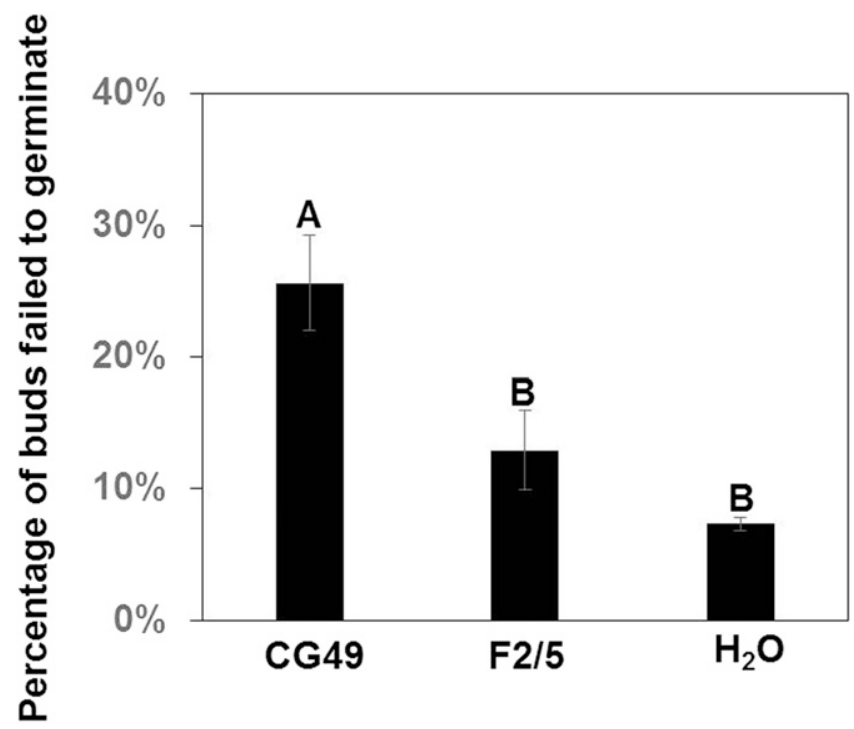

Fig. 4. Impact of CG49 and F2/5 on bud germination of grafted cuttings. Shown are mean percentage of failed bud germination with $\mathrm{CG} 49, \mathrm{~F} 2 / 5$, and $\mathrm{H}_{2} \mathrm{O}$ treatments at 30 days postinoculation. At least 46 samples were included for each treatment and the experiment was repeated three times. Error bars are standard errors. Different letters represent a significant statistical difference by one-tailed Student's $t$ test with a $P<0.05$. 
of the water treatment (Avg $=27.5 \mathrm{~N}, P<0.01)$ (Fig. 7). This was presumably due to the induction of crown gall infections (observed as callus) which, at this point of development, strengthened the graft union. In contrast, F2/5 treatment resulted in lower graft strength $($ Avg $=22 \mathrm{~N})$ than the control $(P<0.01)$. At $60 \mathrm{dpi}$, no significant difference was observed in graft strength between CG49 and the water control whereas F2/5-treated plants were lower than both. The differences of strength at $60 \mathrm{dpi}$ showed that water-treated grafts developed the greatest increase in mechanical strength between 30 and 60 days as compared with either of the bacterial treatments (Fig. 7).

\section{Discussion}

A. vitis is randomly distributed throughout dormant grape canes, including in nodal and intermodal tissues (Johnson et al. 2013, 2016); therefore, the bacterium can persist in propagation material used in grafting. In this study, we sought to enhance our understanding of the impacts that $A$. vitis may have on graft union formation and on plant growth.

To facilitate this work, an inoculation method was developed that consists of soaking dormant cuttings in bacterial suspensions prior to making grafting cuts, during which time the bacterium is spread on to the graft union surfaces. During the soaking and inoculation step, living grape cells at the basal and apical cut ends of the cuttings would also come in contact with the bacterium. Therefore, the some of the effects we observed on plants, such as bud germination and shoot biomass, could result from the bacterium affecting graft union formation or from its presence on other surfaces of the cutting. Because A. vitis is endophytic and epiphytic on and in vines and is randomly distributed in dormant cuttings, the method was designed to mimic how wound inoculations may occur during propagation events at sites on the canes hosting natural $A$. vitis cells; we hypothesize that, should a graft or other cut be made through a site naturally colonized by $A$. vitis, the bacterium would multiply on the wound, similarly to what was shown previously on cut ends of shoot explants (Herlache et al. 2001). Indeed, we observed that the bacterium grows rapidly over a short period of time following wounding. Cell numbers increase more than a 1,000-fold within 5 days, and a high population level is maintained at $60 \mathrm{dpi}$. In fact, such bacterial growth was the basis of previous indexing methods used to detect $A$. vitis associated with dormant cuttings (i.e., cuttings were callused and isolations of the bacterium were made from the callus tissue that formed at the base of the cutting) (Burr et al. 1998; Lehoczky 1968). It is worth noting that, in nature, A. vitis is likely to exist at various cell densities at different sites within cuttings. Therefore, this research serves to demonstrate population dynamics of the pathogen at graft and basal cut
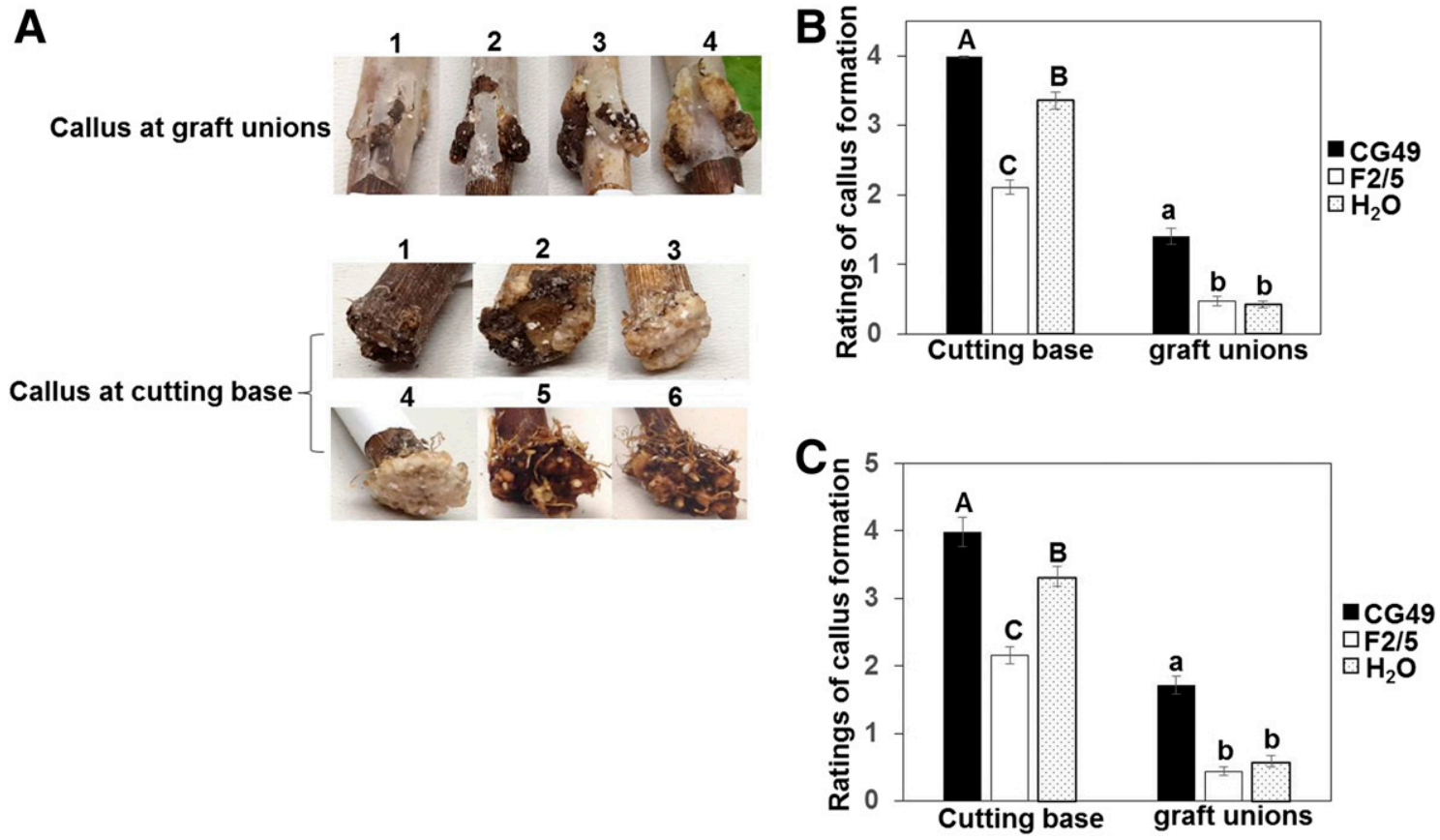

Fig. 5. Callus formation induced by CG49 and F2/5 at graft unions and base of cutting. A, A 1-to-4 or 1-to-6 scaling system for callus formation at graft unions or cutting base, respectively, and mean ratings of callus induced by CG49 (solid filled bars), $\mathrm{F} 2 / 5$ (empty bars), and $\mathrm{H}_{2} \mathrm{O}$ (dotted filled bars) treatments at cutting base and graft unions at B, 30 or $\mathrm{C}$, 60 days postinoculation, respectively. At least 18 samples were included for each treatment at each time point and the experiment was repeated at least three times. Error bars are standard errors. Different letters represent a significant statistical difference with a $P<0.01$ by Tukey's honestly significant difference test.
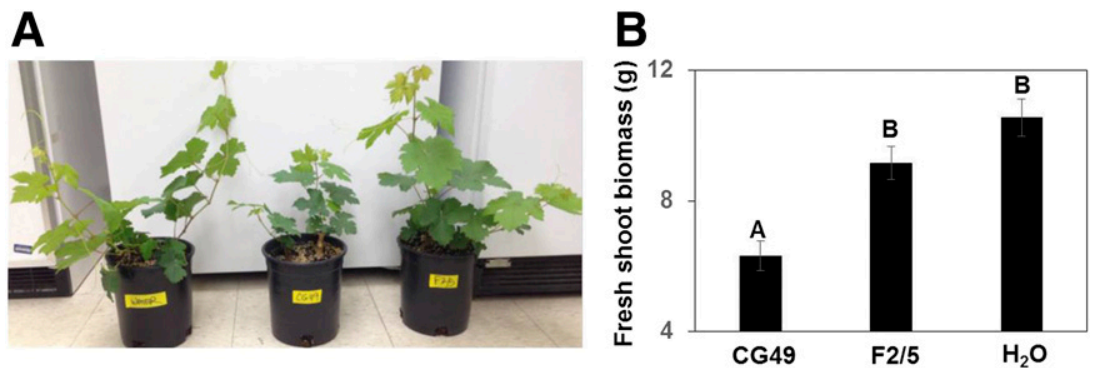

Fig. 6. Shoot development on grafted cuttings inoculated with CG49 and F2/5. A, Representative image of shoot development of plants inoculated with $\mathrm{H}_{2} \mathrm{O}$ (left), $\mathrm{CG} 49$ (middle), and F2/5 (right), and B, mean fresh shoot biomass of grafts inoculated with CG49, F2/5, and $\mathrm{H}_{2} \mathrm{O}$ at 60 days postinoculation. At least 18 samples were included for each treatment and each experiment was repeated at least three times. Error bars are standard errors. Different letters represent a significant statistical difference by Tukey's honestly significant difference test with a $P<0.01$. 
sites and, thus, is informative for furthering our understanding of bacterial ecology in grapevines.

At the graft union, an initial layer consisting of dead cells and associated cell wall materials such as pectin, is deposited that seals off the wounded area from water loss and possibly pathogen infection. Then, callus cells, differentiating from the parenchymatous tissue, start to proliferate and eventually fill the space between the scion and rootstock and, in some cases, rupture the necrotic layer (Melnyk 2017). This wound-induced callusing process involves reprogramming of a large set of plant genes and involves signaling of hormones such as auxin (Cookson et al. 2013). This differs from Agrobacterium sp.-induced callus formation that is dependent on T-DNA transfer and expression in the plant genome (Ikeuchi et al. 2013). Basipetal transport of auxin from buds results in auxin accumulation at wound sites and at basal ends of cuttings and increases cell susceptibility to transformation by $A$. vitis (Creasap et al. 2005). Indeed, CG49 induced strong callus formation (crown gall) at graft unions and at basal ends of the cuttings at $30 \mathrm{dpi}$ (Fig. 5B), which was not seen in the water or F2/5 treatments. In contrast, F2/5 inhibited callus formation at the basal ends, which may be associated with its ability to induce necrosis on grape (Fig. 2B). Necrosis may be causing death of cambial cells that would normally initiate callus formation. A similar trend was observed at 60 dpi (Fig. 5C) and the amount of callus formation was similar between the two time points, which is consistent with a previous report that callus is readily formed in grapevine grafts after approximately 28 days (Cookson et al. 2013). We did not observe an obvious effect of F2/5 on total fresh root biomass when inoculated at the $10^{6} \mathrm{CFU} / \mathrm{ml}$ level. Callusing is important for tissue differentiation and regeneration. However, in most cases, callus formation and root development are independent of each other, although adventitious roots can emerge from callus tissues (Karimi and Yadollahi 2012; Shibuya et al. 2014). Another possibility is that the effect of F2/5 on root development may become more apparent at higher inoculum levels or over longer periods of time. It should be noted that the callus formation we observed at the graft unions was on the external surface because it was not possible to rate internal callus without sacrificing the samples.

We hypothesized that tumorigenicity and necrosis caused by A. vitis are detrimental to graft healing and strength. The few studies which have quantified the effect of specific factors on graft strength have primarily focused on herbaceous plants using very low load (force) regimes. To the best of our knowledge, the only report of graft strength on woody plants was that done on cherry trees (Pedersen 2005). A goal of our research was to develop a method which would quantitatively measure the strength of the graft bond on relatively young woody plants. A graft strength test should be effective over a wide range of loads, accommodate efficient processing of samples, and avoid stress concentrations due to clamping. We examined two testing methods: tension testing as used by Pedersen (2005) and a three-point bending method. During preliminary trials, we found that

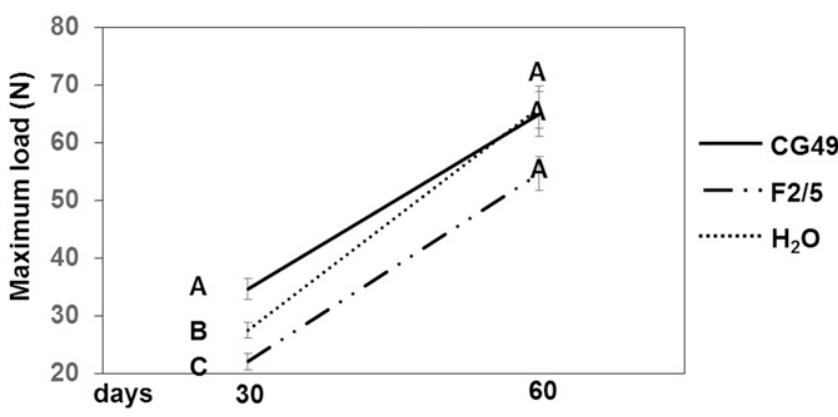

Fig. 7. Strength of grafts inoculated with $\mathrm{CG} 49$ and F2/5 after 30 and 60 days. Shown are mean maximum loads (Newtons) required to break grafts inoculated with CG49 (solid line), $\mathrm{F} 2 / 5$ (dashed line), and $\mathrm{H}_{2} \mathrm{O}$ (dotted line) at 30 or 60 days postinoculation. At least 18 samples were included for each treatment at each time point and each experiment was repeated at least three times. Error bars are standard errors. Different letters represent a significant statistical difference by Tukey's honestly significant difference test with a $P<0.01$. the clamping process associated with tension testing put an unsatisfactory large and uncontrolled preload on the grafted cutting. Because the samples were only carrying loads of 10 to $130 \mathrm{~N}$, it was imperative that the testing began at a true zero load. Any preload would likely damage the graft bond, rendering experiments less accurate. The second method that we investigated, three-point bend testing, had the advantage of loading the sample without restraints. This meant that we had no preload and this allowed us to rapidly process samples (less than $2 \mathrm{~min} / \mathrm{sample}$ ).

We also considered how the graft geometry would affect the graft strength measurement and settled on using a "V" graft, oriented such that the load vector lies within both planes of the "V" graft. This meant that there was minimal geometric resistance to bending, so that any force measured would be due to the regrowth of the vine at the graft. Other geometries such as an " $\Omega$ " graft, would have additional geometric resistance to bending that would be advantageous for graft strength in the nursery but detrimental to measuring strength differences of the new growth across the graft.

Three-point bending is a good test for determining graft strength of woody stems because of practical considerations given above but also because it approximates common failure modes in the field that may be caused by factors such as high winds. This method involves putting one side of the grafted sample in tension while the other side is in compression. A break in the glue-like material (such as callus) will open on the tensile side of the sample. As the graft union matures, fiber formation results in significant strengthening in tension and can carry the load even after the glue has fractured.

For a successful graft to develop, organized, functional vascular bundles need to form. During the phase of rapid callus cell division, an initial adhesion occurs to help hold the graft partners together and provide increased mechanical strength. CG49-treated plants had higher average graft strength at day 30 , which may be associated with the greater callus (crown gall) that could serve as additional "glue." However, crown gall also impairs normal healing and the generation of organized vascular bundles (Gohlke and Deeken 2014), resulting in impaired function, reduced plant vigor, and, subsequently, stunted plants in the long term. Consistently, CG49-treated plants at day 60 developed significantly less fresh shoot biomass than the water control group. F2/5, on the other hand, had less average graft strength at day 30 , presumably due to necrosis at the graft union, which was illustrated in the initial dose effect assay. However, at day 60, F2/5-treated plants showed no apparent difference from the water treatment in fresh shoot biomass but did have reduced graft strength (Fig. 7). Successful grafts continue to strengthen over time; we observed that the water-treated plants showed a greater increase in graft strength from day 30 to 60 as compared with those of CG49- or F2/5treated ones. The necrosis caused by F2/5 in our experiments did not appear to inhibit plant growth; however, it may be that differences would become apparent in longer-term experiments or that the plant is able to compensate for the necrosis as it ages. It has been reported that incompatibility of grafts may be observed in the short or long term (Goldschmidt 2014). Whether F2/5 has a negative impact on graft union development and plant growth at later time points needs to be further investigated. It is worth noting that plants with no root development or bud germination at day 30 were excluded from the evaluations of callus formation, root or shoot biomass, and graft strength analysis, because those plants were unable to grow after being transplanted into the soil. Similarly, plants that declined in growth (bud growth collapsed) after being transplanted into soil were also excluded to avoid bias between time intervals. Combining the data showed that CG49 caused lower levels of bud germination and F2/5 caused increased bud collapse shortly after buds began to grow; therefore, the data present an underestimation of the negative effects on grafts caused by the two strains in these assays. Moreover, we determined that the plant material used in the experiments carried tumorigenic $A$. vitis, which is another factor that likely contributed to the variance among experiments. We attempted to account for this factor by including enough replicates for each experiment, because the natural occurrence of the bacterium in grape cuttings is almost unavoidable due to its systemic nature and random distribution. 
Overall, both the tumorigenic CG49 and nontumorigenic F2/5 strains negatively affected graft union strength and plant growth. It will be important to extend this research to include evaluations of grafted vines over several months in natural environments. In also may be that certain rootstock-scion combinations will be affected differently by $A$. vitis. The information presented enhances our understanding of $A$. vitis interactions with grapevine and will be important for development of strategies to manage crown gall in commercial nurseries and vineyards.

\section{Acknowledgments}

This project was partially funded by the United States Department of Agriculture Federal Capacity Fund grant 2015-16-198 to T. Burr. We thank L. Johnson at Cornell Statistical Consulting Unit for help with statistical analysis and C. Reid and B. Dehaven of Cornell University-New York State Agricultural Experiment Station, Geneva, NY for conducting the A. vitis detection assay and plant and greenhouse management, respectively.

\section{Literature Cited}

Alleweldt, G., and Possingham, J. V. 1988. Progress in grapevine breeding. Theor. Appl. Genet. 75:669-673.

Atkinson, C. J., Else, M. A., Taylor, L., and Dover, C. J. 2003. Root and stem hydraulic conductivity as determinants of growth potential in grafted trees of apple (Malus pumila Mill.). J. Exp. Bot. 54:1221-1229.

Bausher, M. G. 2013. Graft angle and its relationship to tomato plant survival. HortScience 48:34-36.

Burr, T. J., Bazzi, C., Süle, S., and Otten, L. 1998. Crown gall of grape: Biology of Agrobacterium vitis and the development of disease control strategies. Plant Dis. 82:1288-1297.

Burr, T. J., Bishop, A. L., Katz, B. H., Blanchard, L. M., and Bazzi, C. 1987. A root-specific decay of grapevine caused by Agrobacterium tumefaciens and A. radiobacter biovar 3. Phytopathology. 77:1424-1427.

Burr, T. J., and Reid, C. L. 1994. Biological control of grape crown gall with nontumorigenic Agrobacterium vitis strain F2/5. Am. J. Enol. Vitic. 45:213-219.

Chen, F., Guo, Y. B., Wang, J. H., Li, J. Y., and Wang, H. M. 2007. Biological control of grape crown gall by Rahnella aquatilis HX2. Plant Dis. 91:957-963.

Cookson, S. J., Clemente Moreno, M. J., Hevin, C., Nyamba Mendome, L. Z., Delrot, S., Trossat-Magnin, C., and Ollat, N. 2013. Graft union formation in grapevine induces transcriptional changes related to cell wall modification, wounding, hormone signalling, and secondary metabolism. J. Exp. Bot. 64: 2997-3008.

Creasap, J. E., Reid, C. L., Goffinet, M. C., Aloni, R., Ullrich, C., and Burr, T. J. 2005. Effect of wound position, auxin, and Agrobacterium vitis strain F2/5 on wound healing and crown gall in grapevine. Phytopathology 95:362-367.

Dandurishvili, N., Toklikishvili, N., Ovadis, M., Eliashvili, P., Giorgobiani, N., Keshelava, R., Tediashvili, M., Vainstein, A., Khmel, I., Szegedi, E., and Chernin, L. 2011. Broad-range antagonistic rhizobacteria Pseudomonas fluorescens and Serratia plymuthica suppress Agrobacterium crown gall tumours on tomato plants. J. Appl. Microbiol. 110:341-352.

Fourie, P. H., and Halleen, F. 2006. Chemical and biological protection of grapevine propagation material from trunk disease pathogens. Eur. J. Plant Pathol. 116:255-265.

Gohlke, J., and Deeken, R. 2014. Plant responses to Agrobacterium tumefaciens and crown gall development. Front. Plant Sci. 5:155.

Goldschmidt, E. E. 2014. Plant grafting: New mechanisms, evolutionary implications. Front. Plant Sci. 5:727.

Hammami, I., Rhouma, A., Jaouadi, B., Rebai, A., and Nesme, X. 2009. Optimization and biochemical characterization of a bacteriocin from a newly isolated Bacillus subtilis strain 14B for biocontrol of Agrobacterium spp. strains. Lett. Appl. Microbiol. 48:253-260.

Hao, G., Zhang, H., Zheng, D., and Burr, T. J. 2005. luxR homolog avhR in Agrobacterium vitis affects the development of a grape-specific necrosis and a tobacco hypersensitive response. J. Bacteriol. 187:185-192.

Herlache, T. C., Zhang, H. S., Ried, C. L., Carle, S. A., Zheng, D., Basaran, P., Thaker, M., Burr, A. T., and Burr, T. J. 2001. Mutations that affect Agrobacterium vitis-induced grape necrosis also alter its ability to cause a hypersensitive response on tobacco. Phytopathology 91:966-972.
Ikeuchi, M., Sugimoto, K., and Iwase, A. 2013. Plant callus: Mechanisms of induction and repression. Plant Cell 25:3159-3173.

Johnson, K. L., Cronin, H., Reid, C. L., and Burr, T. J. 2016. Distribution of Agrobacterium vitis in grapevines and its relevance to pathogen elimination. Plant Dis. 100:791-796.

Johnson, K. L., Zheng, D., Kaewnum, S., Reid, C. L., and Burr, T. 2013. Development of a magnetic capture hybridization real-time PCR assay for detection of tumorigenic Agrobacterium vitis in grapevines. Phytopathology 103:633-640.

Karimi, S., and Yadollahi, A. 2012. Using putrescine to increase the rooting ability of hardwood cuttings of the peach $\times$ almond hybrid GF677. J. Agrobiol. 29:63-69.

Kawaguchi, A. 2013. Biological control of crown gall on grapevine and root colonization by nonpathogenic Rhizobium vitis strain ARK-1. Microbes Environ. 28:306-311

Kawaguchi, A., and Inoue, K. 2012. New antagonistic strains of non-pathogenic Agrobacterium vitis to control grapevine crown gall. J. Phytopathol. 160:509-518

Kawaguchi, A., Inoue, K., and Ichinose, Y. 2008. Biological control of crown gall of grapevine, rose, and tomato by nonpathogenic Agrobacterium vitis strain VAR03-1. Phytopathology 98:1218-1225.

Lehoczky, J. 1968. Spread of Agrobacterium tumefaciens in the vessels of the grapevine after natural infection. Phytopathology 63:239-246.

Lindsay, D. W., Yeoman, M. M., and Brown, R. 1974. An analysis of the development of the graft union in Lycopersicon esculentum. Ann. Bot. (Lond.) 38:639-646.

Melnyk, C. W. 2017. Plant grafting: Insights into tissue regeneration. Regeneration 4:3-14.

Milien, M., Renault-Spilmont, A.-S., Cookson, S. J., Sarrazin, A., and Verdeil, J.-L. 2012. Visualization of the 3D structure of the graft union of grapevine using X-ray tomography. Sci. Hortic. (Amsterdam) 144:130-140.

Moore, R. 1983. Studies of vegetative compatibility-incompatibility in higher plants. IV. The development of tensile strength in a compatible and an incompatible graft. Am. J. Bot. 70:226-231.

Orel, D. C., Reid, C. L., Fuchs, M., and Burr, T. J. 2017. Identifying environmental sources of Agrobacterium vitis in vineyards and wild grapevines. Am. J. Enol. Vitic. 68:213-217.

Parkinson, M., and Yeoman, M. M. 1982. Graft formation in cultured, explanted internodes. New Phytol. 91:711-719.

Pedersen, B. H. 2005. Development of tensile strength in compatible and incompatible sweet cherry graftings. Can. J. Bot. 83:202-210.

Schell, J., and Van Montagu, M. 1977. The Ti-plasmid of Agrobacterium tumefaciens, a natural vector for introduction of nif genes in plants? Basic Life Sci. 9:159-179.

Shibuya, T., Taniguchi, T., Tsukuda, S., Shiozaki, S., and Itagaki, K. 2014 Adventitious root formation of Japanese cedar (Cryptomeria japonica D. Don) cuttings is stimulated by soaking basal portion of cuttings in warmed water while cooling their apical portion. New For. 45:589-602.

Staphorst, J. L., van Zyl, F. G. H., Strijdom, B. W., and Groenewold, Z. E. 1985. Agrocinproducing pathogenic and nonpathogenic biotype-3 strains of Agrobacterium tumefaciens active against biotype-3 pathogens. Curr. Microbiol. 12:45-52.

Tandonnet, J. P., Cookson, S. J., Vivin, P., and Ollat, N. 2010. Scion genotype controls biomass allocation and root development in grafted grapevine. Aust. J. Grape Wine Res. 16:290-300.

Zarrouk, O., Testillano, P. S., Risueño, M. C., Moreno, M. Á., and Gogorcena, Y. 2010. Changes in cell/tissue organization and peroxidase activity as markers for early detection of graft incompatibility in peach/plum combinations. J. Am. Soc. Hortic. Sci. 135:9-17.

Zheng, D., and Burr, T. J. 2013. An Sfp-type PPTase and associated polyketide and nonribosomal peptide synthases in Agrobacterium vitis are essential for induction of tobacco hypersensitive response and grape necrosis. Mol. PlantMicrobe Interact. 26:812-822.

Zheng, D., and Burr, T. J. 2016. Inhibition of grape crown gall by Agrobacterium vitis $\mathrm{F} 2 / 5$ requires two nonribosomal peptide synthetases and one polyketide synthase. Mol. Plant-Microbe Interact. 29:109-118.

Zheng, D., Hao, G., Cursino, L., Zhang, H., and Burr, T. J. 2012. LhnR and upstream operon LhnABC in Agrobacterium vitis regulate the induction of tobacco hypersensitive responses, grape necrosis and swarming motility. Mol. Plant Pathol. 13:641-652.

Zheng, D., Zhang, H., Carle, S., Hao, G., Holden, M. R., and Burr, T. J. 2003. A luxR homolog, aviR, in Agrobacterium vitis is associated with induction of necrosis on grape and a hypersensitive response on tobacco. Mol. Plant-Microbe Interact. 16:650-658. 